\title{
REVIEW ARTICLE \\ The epidemiology of the outbreak of severe acute respiratory syndrome (SARS) in Hong Kong - what we do know and what we don't
}

\author{
I. T. S. YU ${ }^{1 *}$ AND J. J. Y. SUNG ${ }^{2}$ \\ ${ }^{1}$ Department of Community and Family Medicine and ${ }^{2}$ Department of Medicine and Therapeutics, \\ The Chinese University of Hong Kong, Shatin, Hong Kong SAR, China
}

(Accepted 28 April 2004)

\section{SUMMARY}

Severe acute respiratory syndrome (SARS) struck Hong Kong bitterly in the spring of 2003, infecting 1755 persons and claiming nearly 300 lives. The epidemic was introduced by travellers from southern China, where the disease had originated. It started in late February and lasted until early June. Two notable 'super-spreading' events were reported, one inside a teaching hospital and the other in a private housing estate. Other than in the super-spreading events, the infectivity in the community appeared to be low, and there were few, if any, asymptomatic or subclinical infections. Health-care workers were at particular risk and accounted for $22 \%$ of all probable cases. The main modes of transmission were through droplet spread and close/direct contacts, but situations conducive to aerosol generation appeared to be associated with higher risk. Our review suggests that there are still many unknown factors concerning the mode of transmission and environmental risk that need to be clarified.

\section{INTRODUCTION}

Severe acute respiratory syndrome (SARS) is the first new infectious disease of the 21 st century that has attracted global attention. In an epidemic that affected many parts of the world, Hong Kong was the hardest hit area. It had the highest incidence (attack) rate, with 1755 probable cases [World Health Organization (WHO) definition] in a population of around 6.7 million, and a high case-fatality ratio of $17 \cdot 0 \%$ (299 deaths in 1755 probable cases) [1].

A report from the Centers for Disease Control and Prevention (CDC) placed Hong Kong in the limelight of the international spread of the epidemic [2]. This review of what is known and what is not known about the epidemiology of SARS in Hong Kong will provide guidance on the formulation of future preventive

\footnotetext{
* Author for correspondence: Dr I. T. S. Yu, Department of Community and Family Medicine, 4/F, School of Public Health, Prince of Wales Hospital, Shatin, Hong Kong SAR, China. (Email: iyu@cuhk.edu.hk)
}

strategies. We shall first look at the distribution of the SARS infections in time, place and person, and then review the evidence for various risk factors and modes of transmission.

\section{Time, place and people distributions}

The global epidemic is believed to have started in Guangdong province of southern China as an outbreak of atypical pneumonia. Between mid-November 2002 and mid-January 2003, small outbreaks appeared in several cities near Guangzhou, the provincial capital. A seafood merchant, who travelled to one of these cities (Zhongshan) in mid-January, was admitted to a major teaching hospital in Guangzhou and sparked a major outbreak there in February 2003 [3]. The epidemic in Hong Kong was traceable to a medical professor working in one of the teaching hospitals in Guangzhou, who visited Hong Kong and stayed in a hotel (Hotel X) on 21 February 2003 [2]. The outbreak in this hotel affected 12 hotel guests 


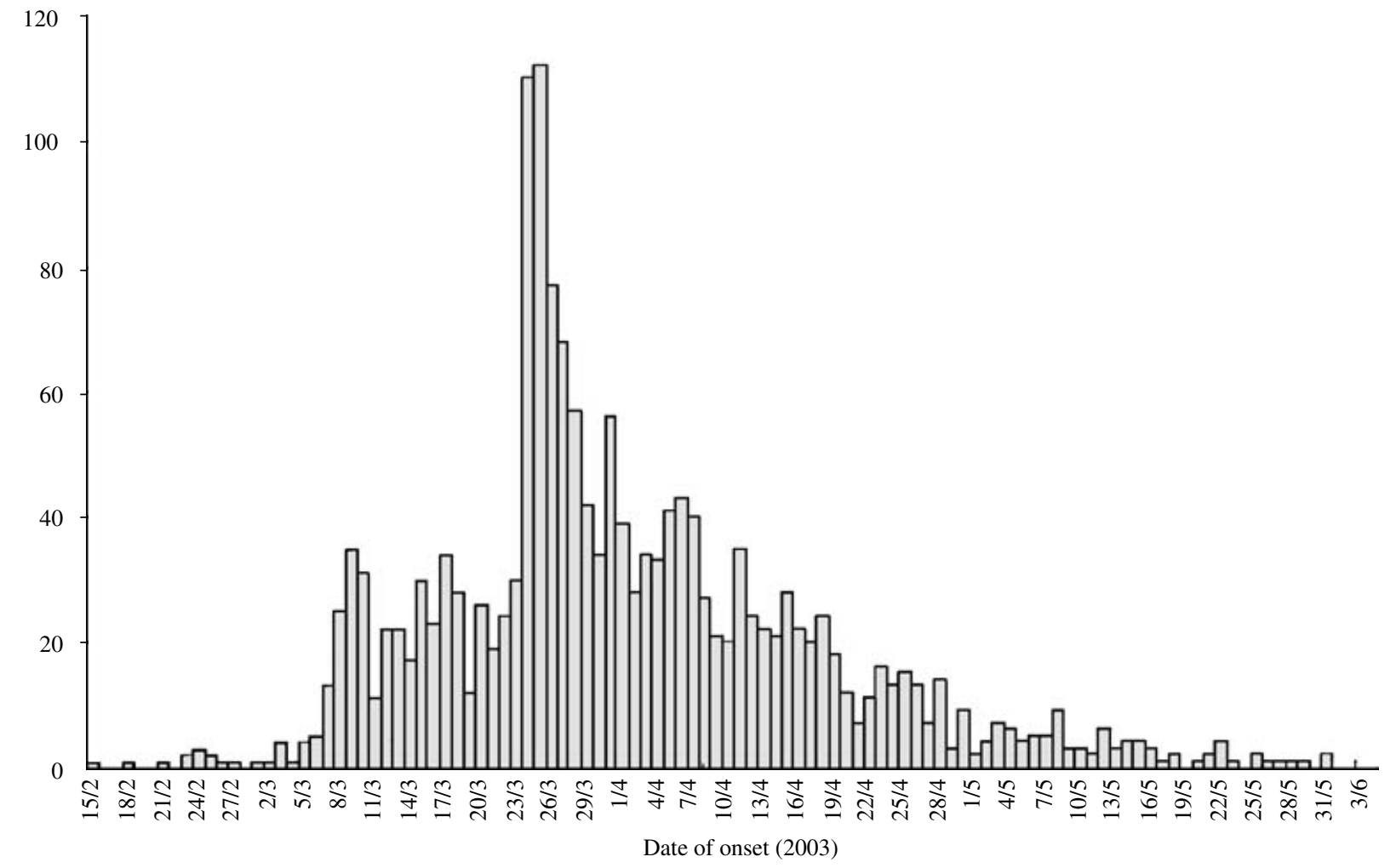

Fig. Epidemic curve of severe acute respiratory syndrome in Hong Kong by date of onset. (Source: SARS Expert Committee. SARS in Hong Kong: from experience to action - Report of the SARS Expert Committee, chapter 3. October 2003.)

(including 2 local guests) and was believed to have led to the subsequent international spread of the disease. A young Hong Kong resident, who visited a friend in Hotel $\mathrm{X}$ in late February, contracted the disease and subsequently gave rise to a large outbreak in the Prince of Wales Hospital (PWH) where he was treated. In the initial phase of the outbreak in the PWH alone, more than 100 persons were affected, including doctors, nurses, medical students, other hospital patients, visitors and their relatives [4]. The report of a government investigation stated that one of the affected patients in the PWH subsequently brought the virus to Amoy Gardens, a private housing estate, where it spread to approximately 300 residents [5].

The epidemic curve of SARS in Hong Kong by date of symptom onset is shown in the Figure. Although the epidemic was believed to have started with the professor from China, several probable cases had presented and been admitted into different Hong Kong hospitals before that. Several peaks were seen in the epidemic curves and are believed to be associated with 'super-spreading' events. The first peak (9-10 March) corresponded to the likely common source outbreak in the PWH. The highest peak (24-28
March) resulted from the massive community outbreak that occurred in Amoy Gardens. After the peak on $25 \mathrm{March}$, the epidemic curve began to decline and there were no new cases reported after 11 June 2003. However, a small peak in early April occurred about a week after the peak admission of residents of Amoy Gardens into other hospitals, suggesting the spread of the disease inside these hospitals.

A substantial proportion of the infections occurred inside public hospitals and the main occupational groups affected were health-care workers; they accounted for $22 \%$ of all probable cases of SARS [6]. It was observed that the cases in the community (excluding Amoy Gardens) tended to cluster around locations of major public hospitals. There was no clear gender preponderance and all age groups were affected except for children who seemed to be less commonly affected, or if infected had a minor illness and better outcome with no mortality [7]. On the other hand, older people could have atypical presentations [8] and had a poorer outcome with high mortality if they contracted the disease [9]. There was no indication that other social demographic factors were important except that medical doctors and 
nurses had a much higher risk compared to others. There were also no data to suggest that genetics, nutritional status, past health, or personal habits played important roles in determining the risk of infection.

\section{Characteristics of the infection}

SARS has an incubation period of 2-16 days [4], with a mean of 6.4 days [9] and a mode of 5 days (based on data from hospital-acquired infections among inpatients in the PWH outbreak and the Amoy Gardens community outbreak). The incubation periods were found to follow a $\gamma$-distribution [9]. There was no evidence that the route of transmission affected the incubation period. The serial interval or generation time was not well documented but appeared to be longer than the incubation period in general. There has also been a lack of good documentation of the duration of infectivity. It appeared that SARS patients were not infectious before the onset of symptoms. The viral load peaked around 10 days after symptom onset [10], and it could be assumed that the peak infectivity also occurred around the same time. Major presenting symptoms included fever, influenza-like symptoms, chills, malaise, loss of appetite, myalgia, cough and headache, but presentations among elderly patients could be quite mild or atypical [8]. Convalescent carriers have not been identified and re-infection among convalescent patients has not been documented, suggesting the presence of some immunity. The lag time from symptom onset to admission varied in the different phases of the epidemic, being longer in the earlier part of the epidemic (average $5 \cdot 36$ days between 26 February and 4 March) and shorter in the later part of the epidemic (average 3.46 days between 9 and 15 April) [9].

The basic reproduction number, $R_{\mathbf{0}}$, defined as the average number of secondary cases generated by one primary case in a susceptible population, was estimated to be around 2.7 without any control measures. This excluded the so-called 'super-spreading' events [11]. Attack rates in community contacts of probable SARS cases were low, estimated as $1.2 \%$ among 19386 family and social contacts under surveillance and $2 \cdot 4 \%$ among the subset of 1158 household contacts on home confinement. The infectivity was fairly low in the general community, but the pathogenicity and virulence appeared to be very high, with only a few reported cases of asymptomatic or subclinical infection [12-14]. The secondary attack rates varied markedly between different cases and settings. Only a small proportion of the symptomatic individuals were infectious (i.e. infected other individuals).

One of the most intriguing characteristics of the 2003 SARS epidemic was the occurrence of 'superspreading' events (SSEs). A SSE referred to a large cluster of infections in which one or more infected individuals disproportionately infected many more individuals than most other infected individuals. The WHO attributed the super-spreading phenomenon to the lack of stringent infection control measures in hospitals during the early days of the epidemic [15], but this could not explain some of the SSEs identified so far, e.g. at Amoy Gardens in Hong Kong. At least two separate SSEs were reported in the Hong Kong epidemic [5, 16]. Additional undocumented SSEs probably existed and it is estimated using mathematical models that approximately $70 \%$ of the infections were attributable to SSEs. SSEs need to be adequately investigated to identify the common underlying factors for effective future prevention. These factors may be associated with the agent (strain), the source (index patient characteristics), the environment (hospital and situational factors) and/or the host (characteristics of the secondary cases). At least two strains of SARS coronavirus were identified from patients in Hong Kong [17] and it is possible that one or more strains were particularly infectious. All probable SARS cases in Hong Kong were believed to result from human-to-human transmission and no animal reservoirs or insect vector were thought to have played any role, though human-to-human transmission did appear to occur through sewerage or some other vehicles in nature. Certain characteristics of the source patient, such as disease severity, virus load, immunodeficiency, atypical presentations, etc., might modify the probability of causing secondary infections. Environmental factors, such as proximity of contact, ventilation and procedures involving aerosolization, were also believed to play important roles in the spread of the disease.

The survival of the SARS-associated coronavirus (SARS Co-V) in different mediums and under different conditions has been studied. The virus was stable in faeces and urine at room temperature for at least 2 days and could survive up to 4 days in stools from patients with diarrhoea because of its higher $\mathrm{pH}$ compared to normal stool [18]. The SARS Co-V has been isolated from stool on paper, a Formica surface and a plastered wall after $36 \mathrm{~h}$, from plastic and stainless-steel surfaces after $72 \mathrm{~h}$, and from a glass slide after $96 \mathrm{~h}[18,19]$. Nothing much is known about 
the antigenic stability, but genetic mutations did occur [17]. Despite the fairly long survival of the virus outside the human body and the possible genetic mutations, disinfection and infection control measures inside hospitals (including triage, isolation of probable cases and cohorting suspected cases) and public health interventions (surveillance, quarantine, environmental decontamination, etc.) in the community eventually appeared to be effective in controlling the epidemic.

\section{Risk factors, environmental factors or conditions}

Other than the inherent host factors mentioned above, certain behavioural, environmental or situational factors could have contributed to infection with SARS. Many of the behavioural factors reported came from anecdotal reports and included rubbing eyes and picking noses with contaminated hands. Case-control studies among health-care workers (and the general population) reported certain behavioural risk/protective factors, such as hand washing and wearing of personal protective clothing [20, 21], but unfortunately the results from these studies could all be explained by recall or reporting bias among cases. Certain high-risk procedures inside hospitals have been reported, including difficult intubations [22], based on the observations of infections of health-care workers after carrying out such procedures or on theoretical grounds. Tomlinson \& Cockram suggested that the use of a nebulizer on a patient with SARS had resulted in the major outbreak in the PWH [16]. However, it was documented later that a substantial number of the infected cases were infected before a nebulizer was used on the index patient [23]. It has also been reported that the use of high flow-rate oxygen masks resulted in health-care worker infections [24].

The variation in secondary attack rates in different settings suggested that environmental factors might be important. A cohort study among medical students in the PWH outbreak suggested that the ventilation system was important in dispersing the infectious agent from an index patient [23]. Analysis of the cohort of patients exposed to the index case in the PWH outbreak (carried out by one of the authors - I.T.S.Y.) also suggested involvement of the ventilation system. Investigation of the Amoy Gardens outbreak implied that the sewage system could act as a mechanical amplifier for the viral source, and poor drainage design and maintenance and the use of strong exhaust fans inside toilets could help to draw the virus from the sewage stack back into the toilets and living areas [5]. Airflows, as determined by temperature, wind direction and configuration of buildings appeared to be important in carrying the virus-laden aerosols to people staying at great distances from the primary source [25].

Most of the available evidence on risk factors is judged to be weak and somewhat indirect according to the commonly accepted hierarchy of evidence. A great deal of work needs to be carried out to separate the essential risk factors from the superfluous ones and to document the relative importance of each true risk factor by estimating the population attributable fraction under different settings - inside hospitals, in the community, etc.

\section{Modes of transmission}

This is one of the most important aspects of SARS infection and needs to be documented as soon as possible if we are to develop rational and effective prevention and control measures against a future return of SARS. There are only a limited number of routes through which an outside agent (biological or chemical) can get inside the human body: inhalation, ingestion, injection or inoculation through the skin, direct contact with the mucous membrane or skin and vertical transmission from mother to foetus through the placenta. There is no evidence that SARS could be transmitted by injection through the skin or from mother to foetus through the placenta [18]. It is also most unlikely that the SARS Co-V could pass through intact skin. There was no documentation of transmission of the SARS Co-V by blood transfusion. Although it was initially speculated that faecal-oral transmission was probably responsible for the mass outbreak in Amoy Gardens, this theory has been largely dismissed by the WHO [18].

The respiratory tract and mucous membranes remain the most likely routes of SARS Co-V entry. The source of the coronavirus for SARS infections in Hong Kong was human SARS patients. The virus has been identified in various body fluids and excretions, including respiratory secretions (nasopharyngeal aspirates and others), blood, urine and faeces. The virus could potentially also be present in tears, saliva and vomitus, and the latter could easily be contaminated by respiratory secretions. There is no evidence to suggest that the virus existed freely in the exhaled air or the desquamated skin. Fomites - inanimate objects or substances that are capable of transmitting infectious organisms from one individual to another - could act 
as an indirect source. Infectious diseases can be transmitted from the source to the recipients through contact spread or a common vehicle (water or food), or can be airborne or vector-borne. There is no evidence to suggest that SARS transmission in Hong Kong was water-, food- or vector-borne, although a hypothesis on the role played by rats has been advocated [26]. Contact spread could be effected through droplets, direct contacts and indirect contacts (fomites), and airborne spread could be through droplet nuclei or virus-laden aerosols of an aerodynamic diameter of $<5 \mu \mathrm{m}$ that could remain suspended in air for a long period of time and inhaled.

Based on clustering of probable cases among close contacts of index patients, it was generally believed that the mode of transmission was mainly through droplet and direct contacts. The evidence is however at best indirect. Having to care for SARS patients and the proximity of contacts have been used as indicators to support transmission by droplet spread and direct contact (through mucous membranes). However, many probable cases of SARS infection contracted inside hospitals did not have direct or close contact (within $1 \mathrm{~m}$ or the range for spread through droplets) with any SARS patient which suggested airborne spread through virus-laden aerosols as a likely mode [23]. Aerosol-generating procedures (use of nebulizer, intubation, etc.) inside hospital have been implicated in a number of nosocomial outbreaks $[16,22]$ and provide further support for airborne spread. Indirect contact through fomites was a distinct possibility due to the long survival of SARS Co-V on inanimate objects. The mass community outbreak in Amoy Gardens, with more than 300 probable cases occurring in several housing blocks tens of metres from one another within a relatively short period of time, could only be adequately explained by airborne spread [25]. As is the case for risk factors, the currently available evidence on the mode of SARS Co-V transmission is weak and indirect. Future laboratory studies will have to be conducted to either confirm or refute different modes of transmission.

\section{Reservoirs}

The last case of SARS in Hong Kong was reported on 11 June 2003. Will SARS return and cause another epidemic in Hong Kong? For SARS to return will need a source or the existence of a reservoir of the SARS Co-V, and suitable environmental conditions for transmission to occur. Studies conducted so far do not support the existence of a human reservoir in Hong Kong [12]. Although viruses related to the SARS $\mathrm{Co}-\mathrm{V}$ have been isolated from wild animals, in particular the masked palm civet and the raccoon dog in southern China [27], there is no evidence of a reservoir in wild or domestic animals found locally in Hong Kong. Introduction of a source from outside Hong Kong is always a possibility, given its close proximity to cities in southern China where the disease originated, and given its nature as an international city with a large volume of travellers in and out of the territory daily. The finding that SARS could be acquired while travelling with symptomatic cases on aircrafts [28] suggested that further efforts should be made to reduce the risk of transmission on different transport vehicles. Vigilance in preventing imported cases must continue and the effectiveness of current border control measures should be properly evaluated.

One must also not forget about the presence of a potential local source-the laboratories that are holding viable cultures of the SARS Co-V. The recent laboratory-associated cases in Singapore and Taiwan $[29,30]$ should have provided ample warning to our researchers and laboratory workers. Most of the population does not have immunity to the disease yet, as the SARS epidemic was prevented from running its natural course in Hong Kong by public health intervention. The large number of susceptible subjects would be a concern should SARS re-emerge, and society should be prepared to take drastic public health measures in order to stop a new infection turning into another epidemic. The recent confirmed case of SARS in Guangzhou [31] should ring an alarm and many questions regarding the source and reservoirs of SARS remain to be answered.

In conclusion, SARS weighed heavily on Hong Kong in 2003, and within a period of several months claimed nearly 300 lives. Despite the huge efforts of the medical profession and researchers, there remains much about SARS that we do not know. Future research must address important issues related to the sources and reservoirs of the virus and also better document the mode(s) of transmission and the important environmental risk factors. Only by doing so can rational and effective preventive and control measures be promulgated in the future.

\section{REFERENCES}

1. World Health Organization. Summary of probable SARS cases with onset of illness from 1 November 2002 
to 31 July 2003 (revised 26 September 2003) (available at: http://www.who.int/csr/sars/country/table2003_09_ 23/en/). Accessed January 2004.

2. Centers for Disease Control and Prevention. CDC update: outbreak of severe acute respiratory syndrome worldwide, 2003. MMWR 2003; 52: 241-248.

3. Zhong NS, Zheng BJ, Li YM, et al. Epidemiology and cause of severe acute respiratory syndrome (SARS) in Guangdong, People's Republic of China, in February, 2003. Lancet 2003 ; 362: 1353-1358.

4. Lee N, Hui D, Wu A, et al. A major outbreak of severe acute respiratory syndrome in Hong Kong. N Engl J Med 2003; 348: 1986-1994.

5. Hong Kong Department of Health. Outbreak of severe acute respiratory syndrome (SARS) at Amoy Gardens, Kowloon Bay, Hong Kong. Main findings of the investigation. 17 April 2003 (available at: http://www.info.gov. hk/info/ap/pdf/amoy_e.pdf). Accessed January 2004.

6. Hong Kong Department of Health. Latest figures on severe acute respiratory syndrome (as at 2 January 2004) (available at: http://www.info.gov.hk/dh/ diseases/ap/eng/infected.htm). Accessed January 2004.

7. Hon KL, Leung CW, Cheng WTF, et al. Clinical presentations and outcome of severe acute respiratory syndrome in children. Lancet 2003; 361: 1701-1703.

8. Ho AS, Sung JJY, Chan-Yeung M. An outbreak of severe acute respiratory syndrome among hospital workers in a community hospital in Hong Kong. Ann Intern Med 2003; 139: 564-567.

9. Donnelly CA, Ghani AC, Leung GM, et al. Epidemiological determinants of spread of causal agent of severe acute respiratory syndrome in Hong Kong. Lancet $2003 ; 361$ : 1761-1766.

10. Peiris JSM, Chu CM, Cheng VCC, et al. Clinical progress and viral load in a community outbreak of coronavirus-associated SARS pneumonia: a prospective study. Lancet $2003 ; 361$ : 1767-1772.

11. Riley S, Fraser C, Donnelly CA, et al. Transmission dynamics of the etiological agent of SARS in Hong Kong: impact of public health interventions. Science 2003; 300: 1961-1966.

12. Chan PKS, Ip M, Ng KC, et al. Severe acute respiratory syndrome-associated coronavirus infection. Emerg Infect Dis 2003; 9: 1453-1454.

13. Lee HKK, Tso EYK, Chau TN, Tsang OTY, Choi KW, Lai TST. Asymptomatic severe acute respiratory syndrome-associated coronavirus infection. Emerg Infect Dis 2003; 9: 1491-1492.

14. Li G, Zhao ZX, Chen LB, Zhou YH. Mild severe acute respiratory syndrome. Emerg Infect Dis 2003; 9: 1182-1183.

15. World Health Organization. Severe Acute Respiratory Syndrome (SARS) - multi-country outbreak - update 30. Status of diagnostic test, significance of 'super spreaders', situation in China, 15 April 2003 (available at: http://www.who.int/csr/don/2003_04_15/en/). Accessed January 2004.

16. Tomlinson B, Cockram C. SARS: experience at Prince of Wales Hospital, Hong Kong. Lancet 2003; 361: 1486-1487.
17. Tsui SKW, Chim SSC, Lo YMD. Coronavirus genomic-sequence variations and the epidemiology of the severe acute respiratory syndrome. N Engl J Med 2003; 349: 187-188.

18. World Health Organization. Consensus document on the epidemiology of severe acute respiratory syndrome (SARS) (available at: http://www.who.int/csr/sars/en/ WHOconsensus.pdf). Accessed January 2004.

19. World Health Organization. First data on stability and resistance of SARS coronavirus compiled by members of WHO laboratory network (available at: http://www. who.int/csr/sars/survival_2003_05_04/en/index.html). Accessed January 2004.

20. Seto WH, Tsang D, Yung RWH, et al. Effectiveness of precautions against droplets and contact in prevention of nosocomial transmission of severe acute respiratory syndrome (SARS). Lancet 2003; 361 : 1519-1520.

21. Lau JTF, Fung KS, Wong TW, et al. SARS transmission among hospital workers in Hong Kong. Emerg Infect Dis 2004; 10: 280-286.

22. Ho PL. Risk factors for SARS in health care workers following intubation of SARS patients-a retrospective multi centre study. SARS Clinical Management Workshop, 13-14 June 2003, China, Hong Kong SAR.

23. Wong T-W, Lee C-K, Tam W, et al. A cluster of severe acute respiratory syndrome among medical students exposed to a single patient in Hong Kong. Emerg Infect Dis $2004 ; 10: 269-276$.

24. Oriental Daily News. 16 May 2003 [in Chinese] (available at: http://orientaldaily.com.hk/cgi-bin/nsrch.cgi? seq = 316968). Accessed January 2004.

25. Yu ITS, Li YG, Wong TW, et al. Probable airborne transmission of severe acute respiratory syndrome (SARS) in the largest community outbreak. N Engl J Med 2004; 350: 1731-1739.

26. Ng SKC. Possible role of an animal vector in the SARS outbreak at Amoy Gardens. Lancet 2003; 362: 570 572 .

27. Guan Y, Zheng BJ, He YQ, et al. Isolation and characterization of viruses related to the SARS coronavirus from animals in southern China. Science 2003; 302: 276-278.

28. Olsen SJ, Chang HL, Cheung TY, et al. Transmission of the severe acute respiratory syndrome on aircraft. N Engl J Med 2003; 349: 2416-2422.

29. World Health Organization. Severe acute respiratory syndrome (SARS) in Singapore - update 2: SARS case in Singapore linked to accidental laboratory contamination (24 September 2003) (available at: http://www. who.int/csr/don/2003_09_24/en/). Accessed January 2004.

30. World Health Organization. Severe Acute Respiratory Syndrome (SARS) in Taiwan, China (17 December 2003) (available at: http://www.who.int/csr/don/2003_ 12_17/en/). Accessed January 2004.

31. World Health Organization. Laboratory confirmation of a SARS case in southern China (5 January 2004) (available at: http://www.who.int/csr/don/2004_01_05/ en/). Accessed January 2004. 